\title{
Materiales híbridos basados en fosfato de vanadilo y polímeros conductores como cátodos en baterias reversibles de litio
}

\author{
A.K. CUENTAS-GALLEGOS, R. VIJAYARAGHAVAN, M. LIRA-CANTÚ, N. CASAÑ-PASTOR, P. GÓMEZ-ROMERO* \\ Instituto de Ciencia de Materiales de Barcelona (ICMAB-CSIC).
}

\begin{abstract}
El fosfato de vanadilo es bien conocido como una fase inorgánica laminar capaz de intercalar una gran variedad de moléculas orgánicas. Mediante la polimerización in-situ, de anilina o pirrol, en la fase inorgánica es posible preparar materiales híbridos orgánico-inorgánicos en los que el polímero queda intercalado. Se describe la síntesis y caracterización de este tipo de híbridos y algunos resultados preliminares de su aplicación como materiales activos de cátodo en celdas reversibles de litio.
\end{abstract}

Palabras clave: Materiales hibridos, baterías de litio, polímeros conductores, fosfato de vanadilo, polianilina, polipirrol, electrodos de inserción de litio.

\section{Hybrid Materials Based on Vanadyl Phosphate and Conducting Polymers as Cathodes in Rechargeable Lithium Batteries.}

Vanadyl phosphate is a well known layered inorganic phase, capable of intercalating a great variety of organic molecules. By means of an in-situ polymerisation of aniline or pyrrole between the layers of the inorganic phase, it is possible to obtain organic-inorganic hybrids. We describe the synthesis and characterization of these type of hybrids, and some preliminary results of their application as active cathodic materials for positive electrodes in reversible lithium cells.

Keywords: Hybrid materials, lithium batteries, conducting polymers, vanadil phosphate, polyaniline, polypyrrole, lithium insertion electrodes.

\section{INTRODUCCIÓN}

Las baterías secundarias de ion litio que están en el mercado emplean óxidos de metales de transición, como electrodos de inserción. Recientemente, los fosfatos de metales de transición han adquirido importancia como materiales viables como cátodos, especialmente donde las limitaciones son cinéticas (1). El fosfato de vanadilo dihidratado ha sido estudiado por Park y colaboradores (2), donde la síntesis seguida fue empleando la sonoquímica (utilizando un equipo de ultrasonidos unidireccional), probándolo como cátodo de inserción de litio. Se obtuvieron muy buenos resultados: una carga específica de $136 \mathrm{mAh} / \mathrm{g}$ y una ciclabilidad estable en 25 ciclos de carga-descarga, además de que la cinética de inserción era casi insensible a la velocidad de carga y descarga entre $0.3 \mathrm{C}$ y $0.03 \mathrm{C}$. El $\mathrm{VOPO}_{4} 2 \mathrm{H}_{2} \mathrm{O}$ tiene una estructura en capas, donde el agua de hidratación se encuentra en el espacio interlaminar. Como huésped el fosfato de vanadilo dihidratado, es capaz de intercalar moléculas, átomos o iones(3). Se han intercalado polímeros conductores como polyipirrol, polianilina, o politiofeno entre otros, por medio de una polimerización oxidativa in-situ en materiales laminares(4), como el $\mathrm{V}_{2} \mathrm{O}_{5}(5-7), \mathrm{SiO}_{2}()$, y $\mathrm{VOPO}_{4} 2 \mathrm{H}_{2} \mathrm{O}$ (8-11). Sin embargo, en el caso del fosfato de vanadilo no han logrado obtener un grado alto de polimerización de la anilina ni una intercalación del polipirrol entre sus capas $(8,10)$. En este trabajo presentamos resultados preliminares de la formación de híbridos mediante la polimerización oxidativa "in-situ" del pirrol y anilina, entre las capas del fosfato de vanadilo dihidratado que se sintetiza mediante un método sonoquímico. Además de la correspondiente caracterización, tanto química como electroquímica para su aplicación como cátodos de inserción de litio.

\section{PARTE EXPERIMENTAL}

\subsection{Síntesis.}

La anilina y el pirrol utilizados para la síntesis de híbridos se pu- rificaron por medio de una destilación a presión reducida, manteniéndolos en atmósfera de $\mathrm{N}_{2}$ a $4^{\circ} \mathrm{C}$. Los ácidos $\mathrm{H}_{3} \mathrm{PO}_{4} 67 \%, \mathrm{HClO}_{4} 70 \%$ y el El $\mathrm{V}_{2} \mathrm{O}_{5} 99.6 \%$ utilizado para la síntesis de $\mathrm{VOPO}_{4} 2 \mathrm{H}_{2} \mathrm{O}$ se usaron sin purificaciones adicionales.

El $\mathrm{VOPO}_{4} 2 \mathrm{H}_{2} \mathrm{O}$ se preparó mediante métodos sonoquímicos siguiendo un procedimiento modificado respecto al publicado en la bibliografía (2). En un matraz Erlenmeyer tapado se mezclaron $2 \mathrm{~g}$ de $\mathrm{V}_{2} \mathrm{O}_{5}, 100 \mathrm{ml}$ de agua desionizada y $31.5 \mathrm{ml} \mathrm{de} \mathrm{H}_{3} \mathrm{PO}_{4}$. La mezcla se trató en un baño de ultrasonidos durante 3 horas, durante las cuales se observó un incremento de la temperatura hasta $40^{\circ} \mathrm{C}$ y la formación de un sólido amarillo. La suspensión se filtró a vacío con un filtro microporoso utilizando membranas millipore tipo $0.45 \mu \mathrm{m}$ (tamaño de partícula muy pequeño), y el sólido se lavó con $10 \mathrm{ml}$ de acetona y se dejó secando en el mismo filtro durante la noche. Este sólido de fórmula $\mathrm{VOPO}_{4} 2 \mathrm{H}_{2} \mathrm{O}$ se utilizó en las síntesis posteriores de los materiales híbridos con anilina y pirrol. La síntesis de los híbridos se llevó a cabo mediante la polimerización oxidativa "in-situ" de la anilina o pirrol, entre las capas del $\mathrm{VOPO}_{4} 2 \mathrm{H}_{2} \mathrm{O}$. Las condiciones de síntesis en el caso del híbrido con anilina, fueron las siguientes: se utilizó una relación de anilina$\mathrm{VOPO}_{4} 2 \mathrm{H}_{2} \mathrm{O}-\mathrm{HClO}_{4}(1: 1: 1)$. La anilina se añadió a una suspensión de $\mathrm{VOPO}_{4} 2 \mathrm{H}_{2} \mathrm{O} / \mathrm{HClO}_{4}$, utilizando una agitación magnética de 500rpm a $0^{\circ} \mathrm{C}$, durante 5 días. $\mathrm{Al}$ término de la síntesis se obtuvo un sólido negro, el cual se filtró y se lavó con agua hasta que el filtrado fue incoloro. El sólido se secó a vacío durante 3 días. Para el caso del híbrido formado con pirrol, las condiciones de síntesis fueron las siguientes: se utilizó una relación de pirrol- $\mathrm{VOPO}_{4} 2 \mathrm{H}_{2} \mathrm{O}-\mathrm{HClO}_{4}$ (1:1:1). En este caso, una mezcla del pirrol con la mitad del ácido total se añadió a una suspensión de $\mathrm{VOPO}_{4} 2 \mathrm{H}_{2} \mathrm{O}$ con la otra mitad de $\mathrm{HClO}_{4}$, utilizando una agitación magnética de $500 \mathrm{rpm}$ a $0^{\circ} \mathrm{C}$, durante 1 hora. $\mathrm{Al}$ término de la síntesis se obtuvo un sólido negro, el cual se filtro y se lavó con agua hasta obtener un filtrado incoloro. El sólido se secó a vacío durante 3 días. 


\subsection{Celda electroquímica}

Para el estudio de las celdas utilizamos montajes compactos y estancos de tipo swagelok, con discos de los materiales de electrodo separados por membranas separadoras de fibra de vidrio impregnada con electrolito. Fabricamos cátodos en polvo de los híbridos utilizando el 70\% de material activo y 30\% de carbono super P. El Li metálico en forma de cinta utilizado como ánodo fue de una pureza del 99.9\%, y el electrolito empleado para la batería fue el comercial "Selectipur" de Merck , consistente en una disolución 1M LiPF 6 en una mezcla 1:1 de carbonato de etilo y carbonato de dimetilo (ethyl carbonate (EC) : dimethyl carbonate (DC) 1:1).

\section{RESULTADOS Y DISCUSIÓN}

Los análisis FTIR se llevaron a cabo en pastillas con $\mathrm{KBr}$, en un espectrofotómetro FTIR Nicolet 710. Los análisis de difracción de rayos-x en polvo se llevaron a cabo en un difractómetro Rigaku Ru-200B de $2 \theta=4$ a 60 con una radiación CuKo. Un sistema Carlo Erba CHN EA 1108 se utilizó para los análisis elementales. Los análisis de SEM se realizaron en un microscopio Hitachi S-570. Para determinar la parte orgánica e inorgánica de los híbridos se utilizó una termobalanza Perkin Elmer ATG-7 con atmósfera de oxígeno; aplicando una rampa lenta de calentamiento de $1^{\circ} \mathrm{C} / \mathrm{min}$ hasta $500^{\circ} \mathrm{C}$, manteniendo la temperatura durante 5 horas para la combustión total del polímero. Para la sínteis sonoquímica del $\mathrm{VOPO}_{4} 2 \mathrm{H}_{2} \mathrm{O}$ se utilizo un baño de ultrasonidos Elma modelo Transonic 460/H. Los estudios electroquímicos (análisis en baterías) se llevaron a cabo en celdas swagelok en un potenciostato multicanal ARBIN BT2042.

\subsection{Caracterización Química}

\section{VOPO $2 \mathrm{H}_{2} \mathrm{O}$}

En la figura 1 presentamos el difractograma de rayos-x que confirma la obtención de la fase $\mathrm{VOPO}_{4} 2 \mathrm{H}_{2} \mathrm{O}$ en las condiciones de nuestro experimento, que representan el uso de una fuente de ultrasonidos de baja potencia. En este difractactograma el pico más relevante es el pico 001 a $2 \theta=11.9$, que representa un espaciado de $7.4 \AA$. Tanto este espaciado como el aspecto general del difractograma coinciden con los datos previamente publicados en la bibliografía $(2,12)$. Sin embargo, en nuestro caso se puede observar un pequeño pico a $2 \theta=6.02$ (espaciado 14.66 ̊̊.) que indicaría la existencia de una superestructura correspondiente al doblado de la celda unidad en la dirección $c$. No obstante, esta pequeña diferencia, aunque significativa desde un punto de vista cristalográfico, no es probablemente de gran relevancia en relación a la obtención de materiales híbridos como los que aquí describimos.

Como parte de caracterización del fosfato de vanadilo dihidratado se llevó a cabo un análisis de espectroscopia infrarroja. Podemos observar el espectro FTIR en la figura 2, donde están marcadas las bandas correspondientes al $\mathrm{VOPO}_{4} 2 \mathrm{H}_{2} \mathrm{O}$, asignadas según la bibliografía (13).

\section{Híbrido PAni/ $\mathrm{VOPO}_{4}$}

La formación del híbrido se comprobó utilizando la espectroscopia infrarroja. En la figura 3 podemos observar el espectro de FTIR del híbrido, donde podemos detectar las bandas correspondientes a la polianilina ( en su forma de poliesmeraldina(14) además de las derivadas del $\mathrm{VOPO}_{4} 2 \mathrm{H}_{2} \mathrm{O}$. Por otra parte, podemos observar que el espectro del material después de un mes de preparado (b) difiere ligeramente del espectro de la muestra recién preparada (a). La muestra envejecida presenta un nuevo pico a $1708 \mathrm{~cm}^{-1}$ que hasta el momento no ha sido asignado.

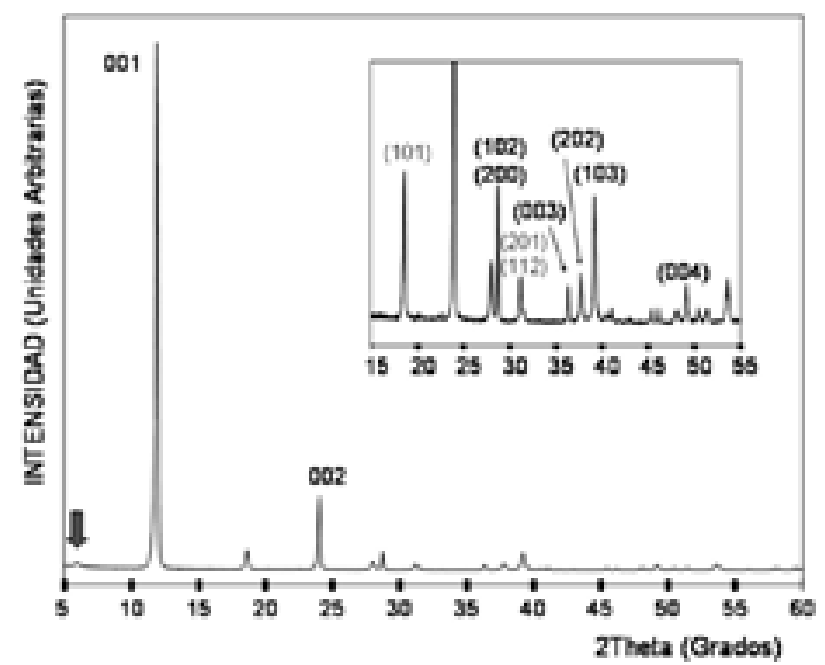

Figura 1.- Difracción de rayos $x$ de $\mathrm{VOPO}_{4} 2 \mathrm{H}_{2} \mathrm{O}$ preparado sonoquímicamente, indexando los picos $(h k l)$ de acuerdo con la bibliografía (2). La flecha marca un débil pico de superestructura (vease texto).

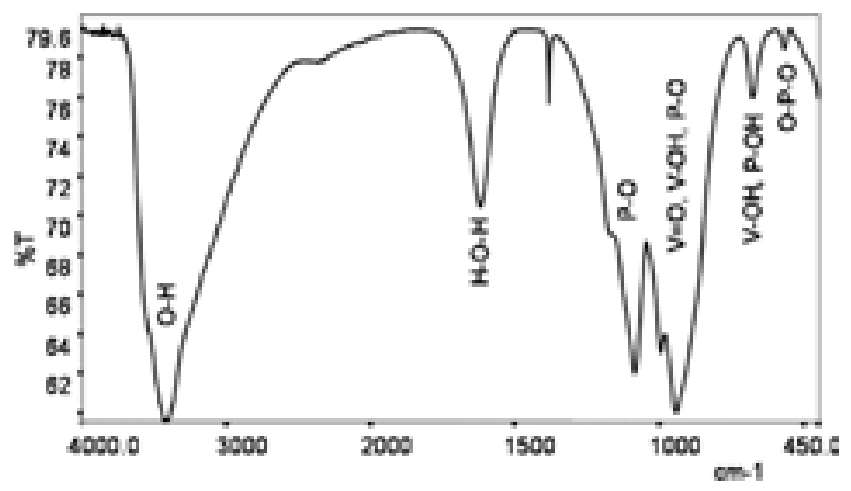

Figura 2.- Espectro infrarrojo del $\mathrm{VOPO}_{4} 2 \mathrm{H}_{2} \mathrm{O}$, donde se indican los modos vibracionales asignados a cada banda.

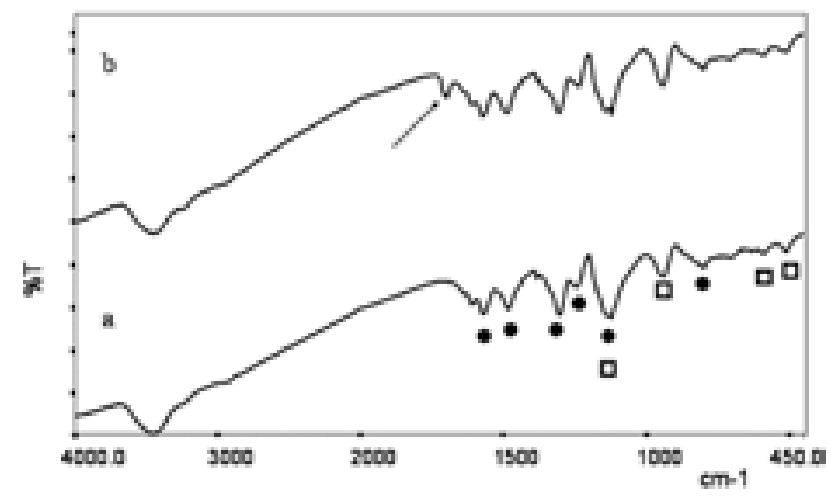

Figura 3.-Análisis FTIR del híbrido PAni/ VOPO . a)espectro del material recién preparado y $b$ ) espectro del material envejecido durante un mes. Los círculos marcan las bandas pertenecientes a la polianilina y los cuadrados al $\mathrm{VOPO}_{4}$. La flecha marca la banda que se intensifica al envejecer el híbrido.

La información sobre la intercalación de la polianilina entre las capas del $\mathrm{VOPO}_{4} 2 \mathrm{H}_{2} \mathrm{O}$ nos la da el difractograma de rayos-x de la figura 4A. Podemos observar que el pico del plano 001, se ha desplazado hacia ángulos mas bajos (de $2 \theta=11.9$ a 7), indicando la expansión entre las capas del $\mathrm{VOPO}_{4} 2 \mathrm{H}_{2} \mathrm{O}$. El espaciado aumenta de $7.4 \AA$ a $12.19 \AA$ indicando la intercalación de una monocapa de polianilina, que concuerda con lo publicado anteriormente. (9). Por otra parte, los picos de difracción son mucho mas anchos y peor definidos en el material híbrido, lo cual indica una disminución sustancial de la cristalinidad. 


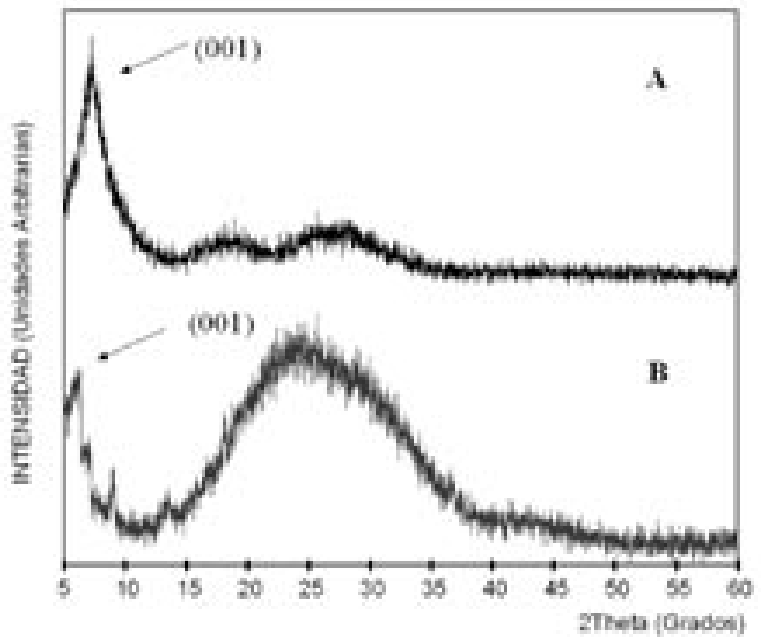

Figura 4.- Difractograma de rayos $x$ del híbrido PAni / $\mathrm{VOPO}_{4}(\mathrm{~A})$ y $\mathrm{PPi} / \mathrm{VOPO}_{4}(\mathrm{~B})$, donde se indica el pico del plano (001) que corresponde al espaciado interlaminar.

En la figura 5a podemos observar la microestructura del híbrido $\mathrm{PAni} / \mathrm{VOPO}_{4^{\prime}}$ donde la escala es de $7 \mu \mathrm{m}$, formada por aglomerados que dan lugar a una estructura microporosa.

Se llevó a cabo un análisis termogravimétrico para determinar la cantidad de materia orgánica en relación al residuo inorgánico del híbrido PAni $/ \mathrm{VOPO}_{4}$. En la figura 6 se presenta la curva de ATG, que indica una pérdida aproximada del $53 \%$ (correspondiente a un residuo del $47 \%$ de materia inorgánica). Inmediatamente después de esta pérdida se detecta un ligero aumento de peso que hemos asignado a la oxidación del V(IV) (producido al inducir la polimerización de la anilina) a $\mathrm{V}(\mathrm{V})$ con absorción de oxígeno. La pérdida del $53 \%$ del peso inicial se da en tres etapas, la primera hasta $120^{\circ} \mathrm{C}$ se asigna a la eliminación del agua de hidratación $(3.86 \%)$, mientras las otras dos etapas deben corresponder a la descomposición del polímero orgánico. Todo ello indica una fórmula de $\left(\mathrm{C}_{6} \mathrm{H}_{4} \mathrm{~N}\right)_{1.9} \mathrm{VOPO}_{4} \cdot 0.73 \mathrm{H}_{2} \mathrm{O}$ para el material híbrido.

\section{Híbrido PPi/ VOPO}

La formación del híbrido se comprobó utilizando la espectroscopia infrarroja. En la figura 7 podemos observar el espectro de FTIR del híbrido recién sintetizado (a) y del mismo material después de un mes de envejecimiento en un desecador(b). Podemos detectar las bandas correspondientes al polipirrol y las pertenecientes al $\mathrm{VOPO}_{4} 2 \mathrm{H}_{2} \mathrm{O}$. Además, de forma similar a lo que ocurría con el híbrido de polianilina, podemos observar que el espectro del material recién sintetizado difiere en una pequeña banda, del espectro de la muestra un mes

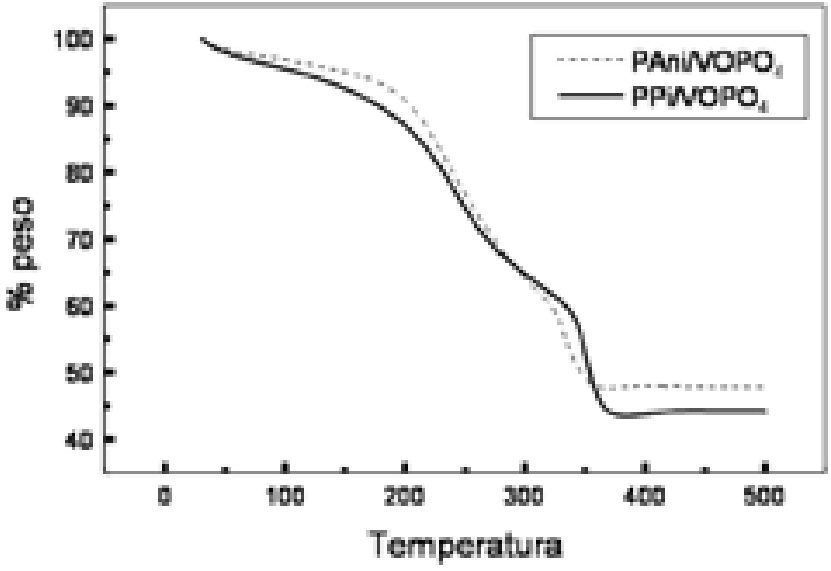

Figura 6.- ATG de las muestras híbridas que se indican, donde se observan los procesos de pérdidas de peso. Condiciones del análisis : $5^{\circ} \mathrm{C} / \mathrm{min}$, hasta $500^{\circ} \mathrm{C}$ durante 5 horas.

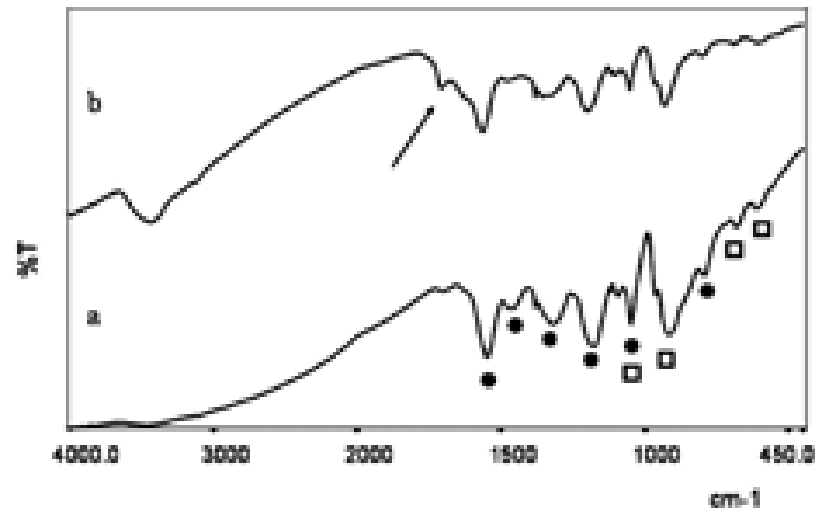

Figura 7.- Análisis FTIR del híbrido $\mathrm{PPi} / \mathrm{VOPO}_{4^{\prime}}$ a) pertenece al espectro del material recién preparado y b) al material envejecido por un mes. Los círculos marcan las bandas pertenecientes al polipirrol y los cuadrados al $\mathrm{VOPO}_{4}$. La flecha indica la banda perteneciente a la sobreoxidación del polipirrol en el híbrido ().

después. En este caso, esta banda alrededor de los $1700 \mathrm{~cm}^{-1}$ que se intensifica en el híbrido de un mes, se ha asociado con la sobreoxidación del pirrol (15).

El difractograma de rayos $x$ del híbrido (figura 4B) nos da algunas de las claves para confirmar la intercalación del polipirrol entre las capas del $\mathrm{VOPO}_{4} 2 \mathrm{H}_{2} \mathrm{O}$. Sobre una base de dispersión difusa característica de los polímeros conductores amorfos y de sus derivados
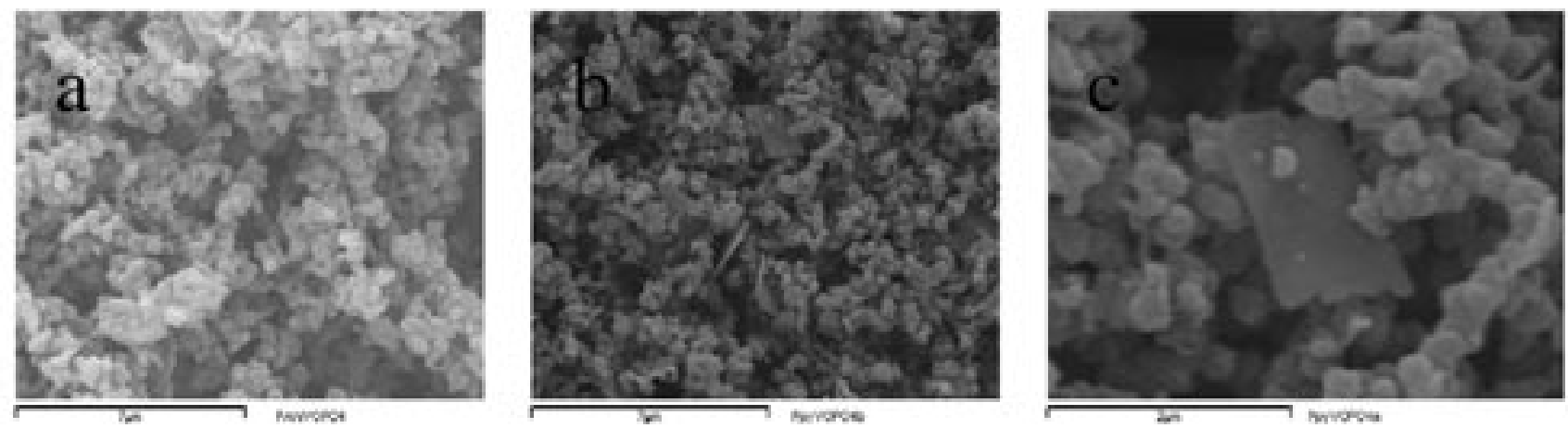

Figura 5.- SEM de los híbridos de $\operatorname{VOPO}_{4} 2 \mathrm{H}_{2} \mathrm{O}$. La foto (a) pertenece al híbrido formado con polianilina (la barra de la escala corresponde a $7 \mu \mathrm{m}$ ), la (b) es del híbrido con polipirrol (escala de $7 \mu \mathrm{m}$ ) y la (c) del híbrido con polipirrol (escala de $2 \mu \mathrm{m}$.) 
híbridos, se pueden observar varios picos débiles entre los que destaca, a ángulos mas bajos, un pico que asignamos como $001 \quad(2 \theta=6)$ que indica la intercalación del polipirrol. Los otros picos más débiles podrían corresponder a la misma fase, o bien indicar la existencia de impurezas de otros intercalatos. Esta segunda hipótesis viene reforzada por la observación mediante microscopía electrónica de barrido de microcristales laminares junto con los aglomerados dominantes en la muestra del híbrido $\mathrm{PPi}_{\mathrm{VOPO}}$ (figura $5 \mathrm{~b}$ y c). Existen 2 tipos de partículas: aglomerados de escala nanométrica correspondientes al propio material híbrido, y plaquitas de $2 \mu \mathrm{m} \times 1 \mu \mathrm{m}$, que se observan mejor en la figura $5 c$, que indican la existencia de pequeñas cantidades de la fase $\mathrm{VOPO}_{4}$ posiblemente intercalada con especies diferentes del propio polímero, y que concuerdan con los resultados obtenidos del análisis DRX.

Se llevó a cabo un análisis termogravimétrico para determinar la cantidad de materia inorgánica y orgánica del híbrido $\mathrm{PPi} / \mathrm{VOPO}_{4}$. En la figura 6 observamos el ATG que es cualitativamente muy similar al observado para el híbrido con polianilina. En este caso el residuo inorgánico corresponde a un $43 \%$ del peso inicial. El restante $57 \%$ perdido, es debido a la eliminación del agua de hidratación (hasta $120^{\circ} \mathrm{C}$ equivalente al 5.5\%) y a la combustión del polipirrol, que indican una fórmula de $\left(\mathrm{C}_{4} \mathrm{H}_{3} \mathrm{~N}\right)_{3} \mathrm{VOPO}_{4} 1.15 \mathrm{H}_{2} \mathrm{O}$. Asimismo, se observa también en este caso la pequeña ganancia de peso por encima de $375^{\circ} \mathrm{C}$ asociada a la oxidación del vanadio.

\subsection{Caracterización electroquímica de los materiales híbridos}

Finalmente hemos llevado a cabo estudios de los materiales híbridos en celdas reversibles frente a ánodos de litio metálico y hemos evaluado parámetros como carga específica y ciclabilidad. El estudio electroquímico se realizó en un rango de voltaje de 3.8-2.1V, aplicando generalmente una corriente de $10 \mathrm{~mA} / \mathrm{g}(20 \mathrm{~mA} / \mathrm{g}$ en un caso). Los datos que se muestran se obtuvieron con celdas en las que los cátodos híbridos se utilizaron en forma de polvo (70\% híbrido y 30\% carbono super $\mathrm{P})$ frente a un ánodo metálico de litio.

$\underline{P_{A n i} / \mathrm{VOPO}_{\underline{4}}}: \mathrm{Al}$ analizar este híbrido como cátodo, se aplicó una corriente de $10 \mathrm{~mA} / \mathrm{g}$ para los primeros cinco ciclos y $20 \mathrm{~mA} / \mathrm{g}$ para los siguientes cinco. La aplicación de distintas corrientes se realizó con la intención de evaluar la cinética de la intercalación de Li. La figura 8A presenta los primeros diez ciclos de carga y descarga del híbrido $\mathrm{PAni} / \mathrm{VOPO}_{4}$. Se puede observar en primer lugar que la carga específica inicial es de $145.3 \mathrm{mAh} / \mathrm{g}$ que es bastante alta. Por otra parte, la inclinación continua de carga y descarga indica un proceso de intercalación en una fase única. Por lo demás se puede apreciar una clara disminución de la carga específica entre el primer ciclo y el segundo, que corresponde a una capacidad irreversible de aproximadamente $50 \mathrm{mAh} / \mathrm{g}$. Esto se expresa mas claramente en la figura $8 \mathrm{~B}$ que recoge los datos de carga específica y capacidad de intercalación de litio en función del número de ciclos. La gráfica de la figura 8B muestra dos aspectos significativos. Por un lado, que la carga específica se mantiene estable en torno a $90-80 \mathrm{mAh} / \mathrm{g}$, y por otro que el paso de un régimen de descarga lento $(10 \mathrm{~mA} / \mathrm{g})$ a otro mas rápido $(20 \mathrm{~mA} / \mathrm{g})$ que tiene lugar en el quinto ciclo representa una mínima disminución en la capacidad del material para almacenar carga. Concretamente se da un cambio de $\approx 89 \mathrm{Ah} / \mathrm{kg}$ a $\approx 72 \mathrm{Ah} / \mathrm{kg}$, es decir una pérdida de un $18 \%$ al doblar el valor de la corriente lo cual representa un buen resultado. Estos resultados indican una buena reversibilidad de los procesos en ambos electrodos, y una cinética rápida para el proceso de intercalación.

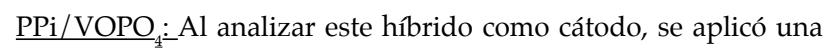
corriente de $10 \mathrm{~mA} / \mathrm{g}$ para los 5 ciclos practicados. En la figura 9 se pueden observar los ciclos sucesivos de carga y descarga del híbrido
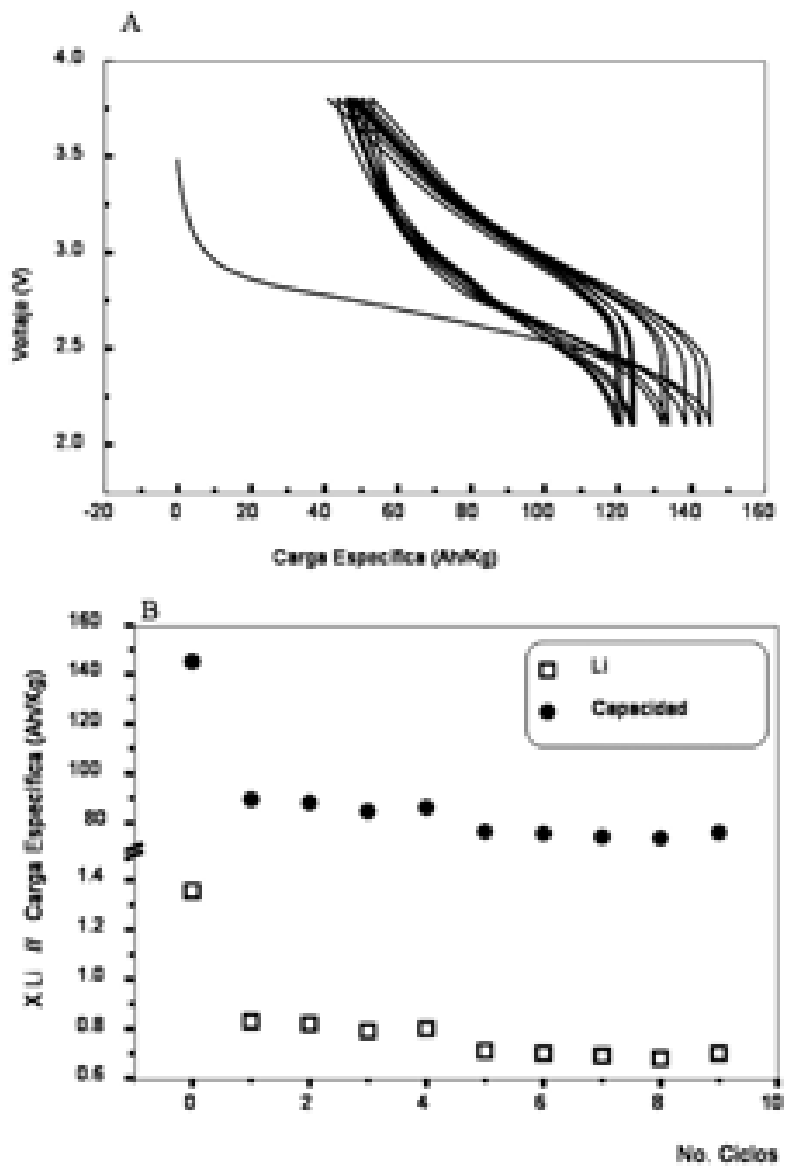

Figura 8.-Análisis electroquímico en baterías de Li del híbrido con polianilina. Se empleó una intensidad de $10 \mathrm{~mA} / \mathrm{g}$ para los primeros cinco ciclos y de $20 \mathrm{~mA}$ /g para los siguientes cinco. A) curvas sucesivas de carga-descarga. B)gráfica donde se representa el valor de la carga específica y del Li intercalado en función del número de ciclos de carga-descarga.

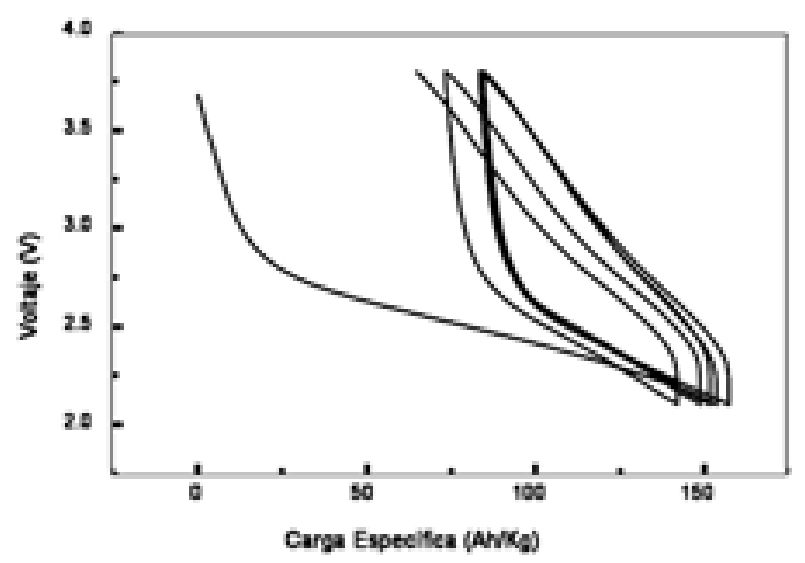

Figura 9.-Análisis electroquímico en baterías de litio del híbrido con polipirrol, $(10 \mathrm{~mA} / \mathrm{g})$ donde se muestran las curvas sucesivas de carga-descarga.

$\mathrm{PPi} / \mathrm{VOPO}_{4}$. La carga específica inicial es de $157 \mathrm{mAh} / \mathrm{g}$ que es relativamente alta, pero la capacidad irreversible es mayor que en el caso del híbrido de polianilina. La carga específica inicial disminuye en el segundo ciclo y se mantiene constante después en torno a $75 \mathrm{mAh} / \mathrm{g}$ indicando una ciclabilidad satisfactoria al igual que ocurría en el caso del híbrido con polianilina. 


\section{CONCLUSIONES}

Hemos preparado la fase $\mathrm{VOPO}_{4} 2 \mathrm{H}_{2} \mathrm{O}$, mediante activación sonoquímica de baja potencia, comprobando su formación por DRX y FTIR. Hemos logrado sintetizar los híbridos derivados de esta fase laminar mediante la formación "in-situ" de polianilina y polipirrol entre las capas del $\mathrm{VOPO}_{4} 2 \mathrm{H}_{2} \mathrm{O}$. Por FTIR comprobamos la formación de los híbridos, detectando las bandas pertenecientes al polipirrol y polianilina, y al $\mathrm{VOPO}_{4}$. Los materiales obtenidos corresponden a las fórmulas preliminares $\left(\mathrm{C}_{6} \mathrm{H}_{4} \mathrm{~N}\right)_{19}$ VOPO $_{4} \cdot 0.73 \mathrm{H}_{2} \mathrm{O}$ y $\left(\mathrm{C}_{4} \mathrm{H}_{3} \mathrm{~N}\right)_{3} \mathrm{VOPO}_{4} \cdot 1.15 \mathrm{H}_{2} \mathrm{O}$ determinadas por ATG. Hemos utilizado estos híbridos como cátodos en celdas reversibles de Li, y muestran una carga específica inicial alta, que baja en el segundo ciclo pero que se mantiene después indicando una buena ciclabilidad. En el caso del híbrido con polianilina hemos podido comprobar que esta buena ciclabilidad es independiente de las corrientes aplicadas, lo cual indica una buena cinética de intercalación del litio.

\section{AGRADECIMIENTOS}

Agradecemos la financiación de este trabajo a través de los proyectos MAT2001-709-C04-01 y MAT2002-04529-C03, y al Consejo Nacional de Ciencia y Tecnología de México (CONACYT) por la concesión de una beca predoctoral a A.KCuentas-Gallegos.

\section{BIBLIOGRAFÍA}

1. T.A. Kerr, J. Gaubicher, y L.F. Nazar, "Highly reversible Li insertion at $4 \mathrm{~V}$ in -VOPO4/_-LiVOPO4 Cathodes", Electrochem. \& Solid State letters., 3, 10, $460-462,2000$.
2. N.G. Park, K.M. Kim, y S.H. Chang, "Sonochemical synthesis of the high energy density cathode material VOPO4.2H2O", Electrochem. Commun. 3 , 553-556, 2001.

3. V. Zima, L. Benes, R. Sisková, P. Fatena, y J. Votinsky, “Intercalation of VOPO4.2H2O with lithium ions", Solid State Ionics, 67, 277-280, 1994.

4. P. Gómez-Romero, "Hybrid organic-inorganic materials. In search of synergic activity", Adv. Mat., 13, 3, 163-174, 2001.

5. S. Kuwabata, S. Masui, H. Tomiyori, y H. Yoneyama, "Charge-discharge properties of chemically prepared composites of $\mathrm{V} 2 \mathrm{O} 5$ and polypyrrole as positive electrode materials in rechargeable Li Batteries", Electrochim. Acta, 46, 91-97, 2000.

6. A.K. Cuentas-Gallegos, M.R. Palacín, M.T. Colomer, J.R. Jurado, y P. Gómez-Romero, "Study of hybrid cathode materials and vitreous anodes. Characterization in lithium ion cells", Bol. Soc. Esp. Ceram. V., 41, 1, 115121,2002.

7. M. Lira-Cantú, P. Gómez-Romero, “Síntesis and characterization of intercalate phases in the organic-inorganic polyaniline/V2O5 system", J. Sol. State Chem., 147, 601-608, 1999

8. N. Kinomura, T. Toyama, y N. Kumada, "Intercalative polymerization of aniline in VOPO4.2H2O", Solid State Ionics, 78, 281-286, 1995

9. H. Nakajima, y G. Matsubayashi, "Intercalation/polymerization of the anilinium Cation in the VOPO4 interlayer space", Chem. Lett., 423-426, 1993.

10. A. De Stefanis, S. Foglia, y A.A.G. Tomlinson, "Assembly and polymerisation of some aromatic amines in _- VOPO4.2H2O", J. Mater. Chem., 5, 3, 475-483, 1995.

11- T. Nakato, Y. Furumi, N. Terao, y T. Okuhara, "Reaction of layered vanadium phosphorus oxides, VOPO4.2H2O and VOHPO4.0.5H2O, with amines and formation of exfoliative intercalation compounds", J. Mater. Chem., 10, 737-743, 2000.

12. Hans R. Tietze, "The Crystal and molecular Structure of oxovanadium (V) orthophosphate dihydrate $\mathrm{VOPO}_{4} .2 \mathrm{H}_{2} \mathrm{O}^{\prime \prime}$, Aust. J. Chem. 34, 10, 2035-2038, 1981.

13. C. R'Kha, M.T. Vandenborre, y J. Livage, “Spectroscopic study of colloidal VOPO $2 \mathrm{H}_{2} \mathrm{O}^{\prime \prime}$, J. Sol. State Chem., 63, 202-215, 1986.

14. T. Fukuda, H. Takezoe, K. Ishikawa, A. Fukuda, H.S. Woo, S.K. Jeong, E.J. Oh, J.S. Suh, "IR and raman studies in three polyanilines with different oxidation levels", Synth. Met., 69, 175-176, 1995.

15. J.B. Schlenoff, y H. Xu, “Evolution of physical and electrochemical properties of polypyrrole during extended oxidation", J. Electrochem. Soc., 139, 9, 2397 2401, 1992.

Recibido: 1.2 .03

Aceptado: 30.11 .03 